\title{
A Robust Sparse Signal Recovery Method for Perturbed Compressed Sensing Based on Max-min Residual Regularization
}

\author{
Rongzong Kang \\ Zhengzhou Information Science \\ and Technology Institute \\ Zhengzhou, China \\ laok_169@163.com
}

\author{
Pengwu Tian \\ Zhengzhou Information Science \\ and Technology Institute \\ Zhengzhou, China
}

\author{
Hongyi Yu \\ Zhengzhou Information Science \\ and Technology Institute \\ Zhengzhou, China
}

\begin{abstract}
Compressive sensing (CS) is a new signal acquisition framework for sparse and compressible signals with a sampling rate much below the Nyquist rate. In this work, we consider the problem of perturbed compressive sensing (CS) with uncertainty in the measurement matrix as well as in the measurements. In order to eliminate the effects of measurement matrix uncertainty, this paper proposed a robust reconstruction method based on max-min residual regularization. We also deduced the solver of the optimization model with the sub-gradient algorithm. Simulation and numerical results shown that the proposed recovery method performs better than the traditional reconstruction methods.
\end{abstract}

Keywords-compressed sensing; max-min; matrix uncertienties; reconstrunction algoritm; analog to information converter(AIC);

\section{INTRODUCTION}

The recently emerged theory of compressed sensing (CS) [1-2] introduced an alternative data acquisition framework to traditional signal acquisition scheme based on ShannonNyquist sampling theory. CS theory enables the acquisition and recovery of sparse signals in some transform domain at a rate proportional to their information content that much below the Nyquist rate.

The problem of reconstructing an unknown sparse vector from a limited number of observations arises throughout engineering and the mathematical sciences. In most applications, these problems are ill-conditioned or underdetermined. Up to now, many mature algorithms have been proposed to resolve this reconstruction problems, including interior-point algorithms [3-4], gradient projection [5], iterative thresholding [6-7], and greedy approaches such as orthogonal matching pursuit (OMP) [8-9]. And these reconstruction algorithms are based on the accurately known dictionary, and most of these recovery algorithms did not considered these uncertainties in the measurement matrix. However in some practices the measurement matrix of compressed sensing may be perturbed. For example, imperfections in the practical signal acquisition hardware, mismatch of system model and parameter discretization, and others.

Some authors have addressed the related problem and analyzed the measurement-matrix uncertainty effect on existing CS reconstruction algorithms. In[10]-[11], the authors analyze the effect of additive perturbations on the
Basis Pursuit and CoSaMP algorithms, In[12] the authors study the worst-case effects on established algorithms. And there are only a few methods that explicitly address measurement-matrix uncertainty. In [13], Zhu et al. extends the classical TLS approach to $\ell_{1}$ regularization, and propose the Sparsity-cognizant Total Least Squares (S-TLS) algorithm. In this paper we propose a robust sparse signal recovery method based on max-min residual regularization for perturbed compressed sensing. Numerical experiments demonstrate that the proposed method has a better performance than the traditional way.

\section{Perturbed Compressed SENSING}

A. The Perturbed compressed sensing System Model

Consider a generalized compressed sensing system, supposed the input signal $x$ is K-sparse. And the measurement matrix is $\boldsymbol{\Phi}$ with $M \times N, M \square N$.the compressed sensing measurements can be expressed as:

$$
\mathbf{y}=\boldsymbol{\Phi} \mathbf{x}
$$

Where $\boldsymbol{\Phi}$ is a $M \times N$ matrix, and it does not depend on the signal $\mathrm{x}$ and it can be constructed as a random matrix such as Gaussian matrix.

However, in practical applications, the measurement vector is often contaminated by noise, thus a perturbed compressed sensing system can be expressed as.

$$
\mathbf{y}=\boldsymbol{\Phi} \mathbf{x}+\mathbf{b}
$$

Where $\mathbf{b}$ denotes the measurement noise.

Furthermore, as we know when measuring an unknown system, in addition to the measurement noise, the sensing matrix may. Thus the perturbed compressed sensing process considering with the perturbed sensing matrix is in the form of

$$
\tilde{\mathbf{y}}=\tilde{\boldsymbol{\Phi}} \mathbf{x}+\mathbf{b}=(\boldsymbol{\Phi}+\mathbf{E}) \mathbf{x}+\mathbf{b}
$$

\section{B. The Perturbed RIP}

However the perturbed matrix $\tilde{\boldsymbol{\Phi}}$ cannot be too dissimilar with the original measurement matrix $\boldsymbol{\Phi}$, it still need to satisfy the conditions of compressed sensing, Here we give the RIP for $\boldsymbol{\Phi}$ [10].

Fix $\mathrm{K}=1,2, \ldots \ldots$ Given the RIC $\delta_{k}$ associated with matrix $\boldsymbol{\Phi}$ and the relative perturbation $\varepsilon_{\mathbf{\Phi}}^{(K)}$ associated with (possibly unknown) matrix $\mathbf{E}$, fix the constant

$$
\hat{\delta}_{K, \max }=\left(1+\delta_{k}\right)\left(1+\varepsilon_{\mathbf{\Phi}}^{(K)}\right)^{2}-1
$$

Then the RIC $\hat{\delta}_{K}$ for matrix $\tilde{\boldsymbol{\Phi}}=\boldsymbol{\Phi}+\mathbf{E}$ is the smallest 
nonnegative number such that

$$
\left(1-\hat{\delta}_{k}\right)\|\mathbf{x}\|_{2}^{2} \leq\|\tilde{\mathbf{\Phi}} \mathbf{x}\|_{2}^{2} \leq\left(1+\hat{\delta}_{k}\right)\|\mathbf{x}\|_{2}^{2}
$$

Holds for any K-sparse vector $\mathbf{x}$ where $\hat{\delta}_{k} \leq \hat{\delta}_{K \text {, max }}$.

\section{Perturbed COMPRESSED SENSING RECOVERY ALGORITHM BASED ON MAX-MIN RESIDUAL REGULARIZATION}

\section{A. The error limits of perturbed compressed sensing}

Here we assume the perturbations to be quantified with the following relative bounds

$$
\frac{\|\mathbf{E}\|_{2}}{\|\mathbf{\Phi}\|_{2}} \leq=\varepsilon_{\boldsymbol{\Phi}}, \frac{\|\mathbf{E}\|_{2}^{(K)}}{\|\boldsymbol{\Phi}\|_{2}^{(K)}} \leq=\varepsilon_{\mathbf{\Phi}}^{(K)}, \frac{\|\mathbf{b}\|_{2}}{\|\mathbf{y}\|_{2}} \leq \varepsilon_{\mathbf{y}}
$$

Where $\|\boldsymbol{\Phi}\|_{2},\|\boldsymbol{\Phi}\|_{2}^{(K)},\|\mathbf{y}\|_{2} \neq 0$ and $\varepsilon_{\boldsymbol{\Phi}}, \varepsilon_{\mathbf{\Phi}}^{(K)}, \varepsilon_{\mathbf{y}}<1$.

While in the practical application we often do not know the exact nature of $\mathbf{E}$ and $\mathbf{b}$.Here we suppose that $\mathbf{E}$ is simply a scaled version of the random measurement matrix $\boldsymbol{\Phi}$, so that $\mathbf{E}=\alpha \boldsymbol{\Phi}$ with $0<\alpha \leq 1$. Denote $\delta_{K}^{\boldsymbol{\Phi}}$ as the RIC associated with the matrix $\boldsymbol{\Phi}$, then for all K-sparse $\mathbf{x}$ the RIP for matrix $\mathbf{E}$ asserts

$$
\alpha^{2}\left(1-\delta_{K}^{\boldsymbol{\Phi}}\right)\|\mathbf{x}\|_{2}^{2} \leq\|\mathbf{E} \mathbf{x}\|_{2}^{2} \leq \alpha^{2}\left(1+\delta_{K}^{\mathbf{\Phi}}\right)\|\mathbf{x}\|_{2}^{2}
$$

Then we can get

$$
\|\mathbf{E}\|_{2}^{(K)} \leq \alpha \sqrt{\left(1+\delta_{K}^{\Phi}\right)}
$$

Thus

$$
\frac{\|\mathbf{E}\|_{2}^{(K)}}{\|\boldsymbol{\Phi}\|_{2}^{(K)}} \leq \alpha \frac{\sqrt{\left(1+\delta_{K}^{\Phi}\right)}}{\sqrt{\left(1-\delta_{K}\right)}}=\varepsilon_{\mathbf{\Phi}}^{(K)}
$$

\section{B. recovery algorithm based on $M M R$}

First we consider the classical compressed sensing problem, given the measurement vector $\mathbf{y}$ and matrix $\boldsymbol{\Phi}$, we need to recover the signal $\mathbf{x}$, we can solve the optimization problem:

$$
\min _{\mathbf{x}}\|\mathbf{x}\|_{1} \text { s.to } \mathbf{y}=\mathbf{\Phi} \mathbf{x}
$$

As we have mentioned before, for the permuted compressed sensing model of(3), if we use the traditional algorithms to recover the sparse signal, it will degrade in the recovery performance. When the error of perturbed compressed sensing is bounded, we can solve the problem as follows:

$$
\begin{aligned}
& \min _{\mathbf{x}}\|\mathbf{x}\|_{1} \quad \text { s.to } \tilde{\mathbf{y}}=\tilde{\boldsymbol{\Phi}} \mathbf{x} \\
& \|\tilde{\boldsymbol{\Phi}}-\boldsymbol{\Phi}\|_{F} \leq \eta,\|\tilde{\mathbf{y}}-\mathbf{y}\|_{2} \leq \eta_{y}
\end{aligned}
$$

For the solution $\hat{\mathbf{x}}$ of each step, we can get the residual error as:

$$
\|(\mathbf{\Phi}+\mathbf{E}) \hat{\mathbf{x}}-(\mathbf{y}+\mathbf{b})\|_{2}
$$

In order to reduce the Min-Max problem to a standard minimization problem, we note that

$$
\min _{\hat{\mathbf{x}}} \max \left\{\begin{array}{l}
\|(\mathbf{\Phi}+\mathbf{E}) \hat{\mathbf{x}}-(\mathbf{y}+\mathbf{b})\|_{2} \\
\|\mathbf{E}\| \leq \eta,\|\mathbf{b}\| \leq \eta_{y}
\end{array}\right.
$$

For

$$
\begin{aligned}
\|(\boldsymbol{\Phi}+\mathbf{E}) \hat{\mathbf{x}}-(\mathbf{y}+b)\|_{2} & \leq\|\boldsymbol{\Phi} \hat{\mathbf{x}}-\mathbf{y}\|_{2}+\|\mathbf{E}\|_{2} \cdot\|\hat{\mathbf{x}}\|_{2}+\|b\|_{2} \\
& \leq\|\boldsymbol{\Phi} \hat{\mathbf{x}}-\mathbf{y}\|_{2}+\eta\|\hat{\mathbf{x}}\|_{2}+\eta_{\mathbf{y}}
\end{aligned}
$$

We therefore conclude that the min-max problem is equivalent to the following minimization problem. Given the measurement matrix $\boldsymbol{\Phi}$, measurements $\mathbf{y}$, and the nonnegative real numbers $\left(\eta, \eta_{\mathbf{y}}\right)$.determine, if possible, an $\hat{\mathbf{x}}$ that solves:

$$
\min _{\hat{\mathbf{x}}}\left(\|\boldsymbol{\Phi} \hat{\mathbf{x}}-\mathbf{y}\|_{2}^{2}+\eta\|\hat{\mathbf{x}}\|_{2}^{2}+\eta_{\mathbf{y}}\right)
$$

Similarly for the K-sparse signal, under the condition of the error of the perturbed compressed sensing system model, we establish the unconstrained optimization problems as follows

$$
\left.\begin{array}{l}
\min _{\hat{\mathbf{x}}}\left(\|\boldsymbol{\Phi} \hat{\mathbf{x}}-\mathbf{y}\|_{2}^{2}+\eta\|\hat{\mathbf{x}}\|_{2}^{2}+\eta_{\mathbf{y}}\right) \\
\min \|\mathbf{x}\|_{1}
\end{array}\right\}
$$

This can be expressed as

$$
\min _{\hat{\mathbf{x}}}\left(\|\boldsymbol{\Phi} \hat{\mathbf{x}}-\mathbf{y}\|_{2}^{2}+\eta\|\hat{\mathbf{x}}\|_{2}^{2}+\eta_{\mathbf{y}}+\|\hat{\mathbf{x}}\|_{1}\right)
$$

Then we define the cost function

$$
\xi(\mathbf{x})=\|\mathbf{\Phi} \mathbf{x}-\mathbf{y}\|_{2}^{2}+\eta\|\mathbf{x}\|_{2}^{2}+\eta_{\mathbf{y}}+\lambda\|\mathbf{x}\|_{1}
$$

This is a convex optimization problem, the first three summands of this cost are differential wrt the optimization variables, while the non-differential third term is separable in the entries of $\mathbf{x}$. And there are many math tools to solve it.

In order to solve the optimization problem in(17), we define the first three summands of this cost as:

$$
\varsigma(\hat{\mathbf{x}})=\|\boldsymbol{\Phi} \hat{\mathbf{x}}-\mathbf{y}\|_{2}^{2}+\eta\|\hat{\mathbf{x}}\|_{2}^{2}+\eta_{\mathbf{y}}
$$

It is obvious that $\varsigma(\hat{\mathbf{x}})$ is a convex continuous function in $\hat{\mathbf{x}}$ and hence any local minimum of $\varsigma(\hat{\mathbf{x}})$ is also a global minimum.

$$
\begin{aligned}
\nabla \varsigma(\mathbf{x}) & =2 \boldsymbol{\Phi}^{T}(\boldsymbol{\Phi} \hat{\mathbf{x}}-\mathbf{y})+2 \eta \hat{\mathbf{x}} \\
& =2\left(\boldsymbol{\Phi}^{T} \boldsymbol{\Phi}+\eta I\right) \hat{\mathbf{x}}-2 \boldsymbol{\Phi}^{T} \mathbf{y}
\end{aligned}
$$

There are many settings for $\mu^{i}$ result in different algorithm involving the gradient method, the steepest descent method, and the Newton's method. In this paper we take the Newton's method for its better convergence performance, that is

$$
\mu^{i}=\left(\boldsymbol{\Phi}^{T} \boldsymbol{\Phi}+\eta I\right)^{-1} / 2(21)
$$

However the last term of the cost function $\|\mathbf{x}\|_{1}$ is not differential when $\mathbf{x}=0$, as a result we define a new subgradient of $x(i)$ entries as follows 


$$
\nabla \xi(\mathbf{x})=\left\{\begin{array}{l}
\nabla \varsigma(\mathbf{x})+\lambda \operatorname{sign}\left(\mathbf{x}_{i}\right),\left|\mathbf{x}_{i}\right| \geq \eta \\
\nabla \varsigma(\mathbf{x})+\lambda,\left|\mathbf{x}_{i}\right|<\eta, \nabla \varsigma(\mathbf{x})<-\lambda \\
\nabla \varsigma(\mathbf{x})-\lambda,\left|\mathbf{x}_{i}\right|<\eta, \nabla \varsigma(\mathbf{x})>\lambda \\
0,\left|\mathbf{x}_{i}\right|<\eta, \nabla \varsigma(\mathbf{x}) \leq \lambda
\end{array}\right.
$$

Then the gradient-based algorithm can be written as

$$
\mathbf{x}(i+1)=\mathbf{x}(i)-\mu^{i} \nabla \xi(\mathbf{x}(i))
$$

\section{Simulation Results}

In order to evaluate the performance of the algorithm we have proposed, numerical simulations were conducted in MATLAB as follows. The reconstruction quality of the algorithm is assessed in terms of the relative recovery error defined as:

$$
S N R=20 \log _{10}\left(\frac{\|\mathbf{x}\|_{2}}{\|\mathbf{x}-\hat{\mathbf{x}}\|_{2}}\right)
$$

In each trial the basis matrix $\boldsymbol{\Psi}$ is the identity matrix, matrix $\boldsymbol{\Phi}$ of size $128 \times 512$ is randomly generated with independent Gaussian distributed entries with zeros mean, in order to normalize the $l_{2}$ norm of each column, the variance is set as $\sigma^{2}=1 / 128$. The nonzero locations of vector $\mathrm{s}$ are randomly selected among all possible choices.

Figure1 shows the reconstruction SNR of the traditional BP method and as a function of different levels of sparsity $\mathrm{K}$ for each $\varepsilon_{E}$. The relative perturbation parameter $\varepsilon_{E}$ are set to $\{0,0.01,0.05,0.1\}$, for each value of $\varepsilon_{E}$ and $\mathrm{K}$, we repeat the simulation for 1000times. As we can see from Fig.1, the reconstruction SNR decrease with the increase of the perturbations parameter $\varepsilon_{E}$, and when $\mathrm{K} K / N=0.045$, the SNR performance has a significant drop, this is because when the measurement $\mathrm{M}$ is fixed, with the increasing number of the sparsity, the RIP condition is no longer to be satisfied, the BP method cannot recover the sparse signal correctly.

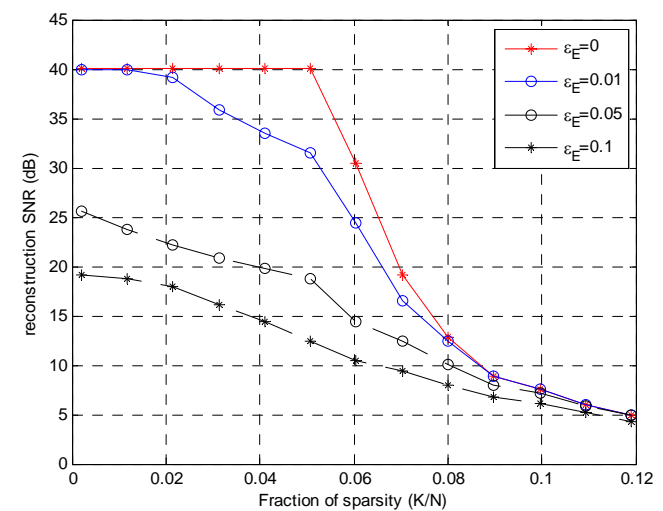

Figure1 reconstruction SNR of BP for different perturbation parameter
Figure 2 shows the Normalized Mean Squared Error (NMSE) of the proposed method compare with the BP algorithm with different level of measurement $\mathrm{M}$. it also give the linear estimates of the true support using $\tilde{\boldsymbol{\Phi}}$. As we can see from the figure2, the proposed method outperforms the BP algorithm. And because the perturbed measurement matrix, the classical BP method cannot achieve estimated values of the true support. Furthermore the NMSE of all the method decrease with the increase of the measurement number $\mathrm{M}$, this is coincide with the compressed sensing theory.

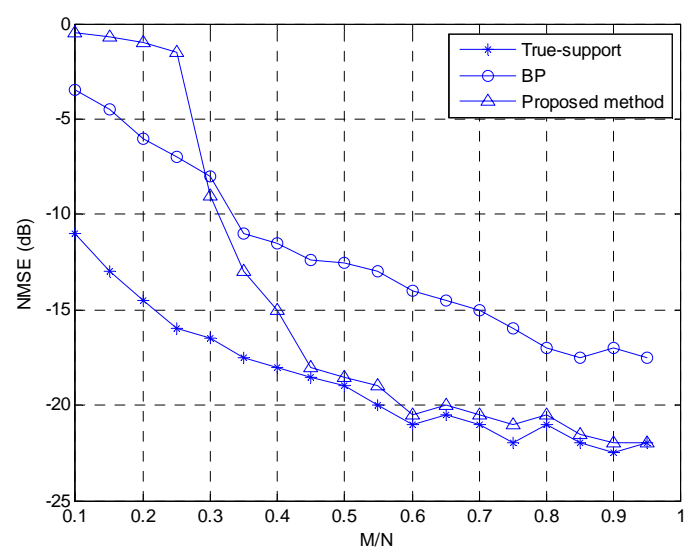

Figure2 the NMSE performance of the reconstruction method with different measurement number $\mathrm{M}$

\section{CONCLUSIONS}

In this paper, the reconstruction problem of perturbed compressed sensing is discussed. We propose a new robust recovery method based on max-min residual regularization. The solver of this optimization problem is also deduced based on the sub-gradient algorithm. The reconstruction SNR of the classical BP algorithm with different perturbation parameter is analyzed; numerical simulations verify that the relative recovery errors of the BP algorithms are linear in the perturbations. The simulations also showed our approaches performed better than the classical BP algorithms in RMSE of signal amplitude recovery. And for the perturbed compressed sensing situation, the proposed method can be a good candidate of compressed sensing recovery algorithms.

\section{REFERENCES}

[1] E. Candes, J. Romberg, and T. Tao, "Quantitative robust uncertainty principles and optimally sparse decompositions," Foundations of Computational Mathematics, November 2004, preprint.

[2] D. Donoho, "Compressed sensing," IEEE Trans. Info. Theory, vol. 52, no. 4, pp. 1289-1306, September 2006.

[3] S. S. Chen, D. L. Donoho, and M. A. Saunders, "Atomic decomposition by basis pursuit," SIAM J. Sci. Comput., vol. 20, no. 1, pp. 33-61, 1999. 
[4] E. van den Berg and M. P. Friedlander, "Probing the Pareto Frontier for basis pursuit solutions,” SIAM J. Sci. Comput., vol. 31, no. 2, pp.890-912, 2008.

[5] M. A. T. Figueiredo, R. D. Nowak, and S. J. Wright, “Gradient projection for sparse reconstruction: Application to compressed sensing and other inverse problems,” IEEE J. Sel. Topics Signal Process., vol. 1,no. 4, pp. 586-597, 2007.

[6] I. Daubechies, M. Defrise, and C. D. Mol, “An iterative thresholding algorithm for linear inverse problems with a sparsity constraint," Commun. Pure Appl. Math., vol. 57, pp. 1413-1457, 2004.

[7] T. Blumensath, M.E. Davies, "Iterative hard thresholding for compressed sensing,” Appl. Comput. Harmon. Anal. vol. 27,no. 3, pp. 265-274,2009.

[8] Y. C. Pati, R. Rezaiifar, and P. S. Krishnaprasad, "Orthogonal matching pursuit: Recursive function approximation with applications to wavelet decomposition,” in Proc. Asilomar Conf. Signals, Syst. Comput., Nov.1993, pp. 40-44.
[9] J. Tropp, A.C. Gilbert, "Signal recovery from partial information via orthogonal matching pursuit," IEEE Trans. Inform. Theory, vol. 53,pp. 4655-4666,2007.

[10] M. A. Herman and T. Strohmer, "General deviants: an analysis of perturbations in compressive sensing," IEEE Journal of Selected Topics in Signal Processing, vol. 4, no. 2, pp. 342-349, 2010.

[11] Y. Chi, A. Pezeshki, L. Scharf, and R. Calderbank, "Sensitivity to basis mismatch in compressed sensing," International Conference on Acoustics,Speech, and Signal Processing (ICASSP 2010), Dallas, TX, Mar. 14-19,2010.

[12] D. H. Chae, P. Sadeghi, and R. A. Kennedy, "Effects of basismismatch in compressive sampling of continuous sinusoidal signals," 2nd Inter-national Conference on Future Computer and Communication, Wuhan, China, May 21-24, 2010.

[13] H. Zhu, G. Leus, and G. Giannakis, "Sparsity-cognizant total leastsquares for perturbed compressive sampling," Signal Processing, IEEE Transactions on, vol. 59, no. 5, pp. 2002 -2016, May 2011. 